Research Article

\title{
Drivers of Stock Prices in Ghana: An Empirical Mode Decomposition Approach
}

\author{
Emmanuel. N. Gyamfi $\mathbb{D}^{1},{ }^{1}$ Frederick A. A. Sarpong $\mathbb{D},{ }^{1}$ and Anokye M. Adam $\mathbb{D}^{2}$ \\ ${ }^{1}$ School of Business, Ghana Institute of Management and Public Administration, Accra, Ghana \\ ${ }^{2}$ School of Business, University of Cape Coast, Cape Coast, Ghana \\ Correspondence should be addressed to Emmanuel. N. Gyamfi; engyamfi@gimpa.edu.gh
}

Received 22 July 2021; Accepted 28 August 2021; Published 26 September 2021

Academic Editor: Yuxing Li

Copyright (c) 2021 Emmanuel. N. Gyamfi et al. This is an open access article distributed under the Creative Commons Attribution License, which permits unrestricted use, distribution, and reproduction in any medium, provided the original work is properly cited.

\begin{abstract}
This study utilized the empirical mode decomposition (EMD) technique and examined which group of investors based on their trading frequencies influence stock prices in Ghana. We applied this technique to a dataset of daily closing prices of GSE Financial Stock Index for the period 04/01/2011 to 28/08/2015. The daily closing prices were decomposed into six intrinsic mode functions (IMFs) and a residue. We used the hierarchical clustering method to reconstruct the IMFs into high frequency, low frequency, and trend components. Using statistical measures such as Pearson product moment correlation coefficient and the Kendall rank correlation, we found that the low frequency and trend components of stock prices are the main drivers of the GSE stock index. These low-frequency traders are the institutional investors. Therefore, stock prices on the GSE are affected by real economic growth but not short-lived market fluctuations.
\end{abstract}

\section{Introduction}

The analysis of financial time series remains a challenging task because of its extremely complex nonlinear and nonstationarity dynamic behaviour [1]. For stock market prices, several factors such as business cycle, government policies, types of investors, and firm-specific factors deepen its complexity [2]. The behaviour of stock prices to quickly discover market imperfections, exploited and corrected by market participant, makes use of conventional time-domain tools inappropriate in analyzing stock markets [3]. For example, the dynamic low-frequency traders like institutional and central banks bringing to the market price formation differ from high-frequency traders like speculators and market makers. This shows that price formation in the stock market is determined by the diverse traders with different trading frequencies. Consequently, some attractive characteristics may not be uncovered if stock prices are analyzed within the time-domain framework. Owing to these, a number of methods have been used in the analysis of stock prices, in general, ranging from linear and nonlinear methods in the frequency-domain $[4,5]$.
In an attempt to solve the problem of nonlinearity and nonstationary in time series data, Huang et al. [6] proposed a time-frequency method named Hilbert-Huang transform (HHT) which is popular in signal analysis $[7,8]$. The technique works through performing a time adaptive decomposition operation named empirical mode decomposition (EMD) on the signal, and then the signal will be decomposed into a set of complete and almost orthogonal components named intrinsic mode function (IMF), which is almost monocomponent. It has been increasingly recognized as an effective analysis model in financial time series. The application of EMD has already been explored in the analysis of economic and financial data. For instance, it has been applied in modelling crude oil price [9-11], exchange rate [12-15], electricity price $[16,17]$, gold price $[18,19]$, and carbon price [20].

In contrast to previous methods such as wavelet analysis and spectrum analysis, EMD is intuitive, direct, posterior, and adaptive [21]. The EMD can be used to decompose a series into a finite and often small number of intrinsic mode functions (IMFs) [6]. 
Similar to EMD, wavelet has been used to study nonstationary signal analysis. Studies such as Tiwari et al. [22, 23]; Jammazi et al. [24]; Jiang et al. [25]; Ferrer et al. [26]; Yang et al. [27]; Wang et al. [28]; Boubaker and Raza [29]; and Frimpong et al. [30] used wavelet-based methods to study the behaviour of financial variables such as oil prices, exchange rates, inflation, and stock prices at different frequencies. However, wavelet has certain drawbacks which EMDs can easily overcome. For instance, wavelet analysis restricts one from selecting an objective wavelet function from a set of wavelet functions. In a wavelet, choosing the basis function and decomposition levels is subjective, and this may lead to the extraction of false cycles forming the time series [31, 32]. EMD nevertheless does not require a subjective test since the decomposition is based on the local characteristic time scale of the data. However, EMD has not been widely used in the analysis of stock prices. Some of the recent studies which have used EMDs in analyzing stock prices are [21, 33-35].

In this paper, we apply EMD in time-frequency analysis for GSE index for the period from 04/01/2011 to 28/08/2015. The main objective of this study is to determine which group of investors based on their trading frequencies influence stock prices in Ghana.

It remains imperative to detect the frequencies that have a significant impact on stock prices. Generally, the stock market reflects the economic conditions of an economy. In a growing economy, there is an increase in output leading to firms experiencing high profitability. These higher profits make shares of companies more attractive because they are able to pay higher dividends to shareholders. A long period of economic growth tends to benefit shareholders.

This study is innovative in the sense that it investigates the group of investors that drive stock prices on the Ghana stock exchange using the empirical mode decomposition (EMD). The following contributions are also made to the literature. First, it provides a detailed analysis of the use of EMD to decompose the stock price into several IMFs and one residue.

Second, these IMFs and the residue are reconstructed into high frequency, low frequency, and trend components using the hierarchical clustering method. Different measures were then used to assess the importance of each frequency for the overall stock price series.

Finally, the economic significance of these components is identified as short-term fluctuations and long-term trend. If the effect of the low frequency or trend components of stock prices on the aggregate stock prices is dominant, it can be concluded that stock prices are driven by the real growth of the economy.

The remainder of the paper is structured as follows. Section 2 provides a brief description of the data. Section 3 describes the methodology. Section 4 contains the results of our analysis, and Section 5 concludes the paper.

\section{Data}

The data which were obtained from DataStream are the daily closing prices of the GSE Financial Stock (GSE-FSI) index coded in DataStream as GSEFSII (Price Index). The data period is between January 4, 2011, and August 28, 2015. The period of study was chosen because of data availability. The closing prices were transformed into returns which were calculated by taking the natural log first difference of prices.

\section{Methodology}

We follow Zhu et al. [20] in presenting the EMD algorithm. The EMD technique assumes that the stock prices of GSEFSII have many IMFs of different oscillations and that an IMF must satisfy two conditions according to Huang et al. [6] as follows:

(i) An IMF has the same number of extremum and zero crossing or differs by one at the most

(ii) An IMF is symmetric with the local zero mean

(iii) To extract IMFs, EMD uses the following sifting process:

(a) All the maxima and minima of the time series (stock prices of GSEFSII) denoted $x(t)$ were identified

(b) Next, we generated upper and lower envelopes $e_{\max }(t)$ and $e_{\min }(t)$ using cubic spline interpolation

(c) The point-by-point mean denoted $m(t)$ were calculated from the upper and lower envelopes as

$$
m(t)=\frac{e_{\max }(t)+e_{\min }(t)}{2} .
$$

(d) The mean was extracted from $x(t)$ and we defined the difference between $x(t)$ and $m(t)$ as $d(t)$. Thus $d(t)=x(t)-m(t)$.

(e) The properties of $d(t)$ were checked. If $d(t)$ was found to be an IMF, we denoted $d(t)$ as the ith IMF and replaced $x(t)$ with the residue; $r(t)=x(t)-d(t)$. The $i$ th IMF is often denoted as $c_{i}(t)$ where $i$ is its index. However, if $d(t)$ was not an IMF, $x(t)$ was replaced with $d(t)$.

Steps (a)-(e) were repeated until the residue satisfied some stopping criteria. See Zhu et al. [20] for more on the stopping criteria.

Therefore, the stock price $x(t)$ can be expressed as the sum of IMFs and a residue. This can be mathematically represented as

$$
x(t)=\sum_{i=1}^{m} c_{i}(t)+r_{m}(t),
$$

where $m$ is the number of IMFs, $r_{m}(t)$ is the final residue, and $c_{i}(t)$ is the $i$ th IMF. 


\section{Results and Discussion}

A plot of the natural logarithmic values of the daily closing prices of GSEFSII for the period from 04/1/2011 to $28 / 08 / 2015$ is shown in Figure 1. Using the empirical mode decomposition (EMD) method, the natural logarithmic values of the prices were decomposed into six different frequencies called the intrinsic mode functions (IMFs) and one residue. The IMFs as plotted in Figure 2 are arranged from high to low frequencies.

Next, the IMFs are analyzed using the following measures: first, we recorded the mean period of each IMF. The mean period is measured as the length of an IMF divided by the number of peaks.

Subsequently, the Pearson product moment correlation coefficient and the Kendall rank correlation coefficient were used to measure correlations between each IMF (residue) and the observed stock prices.

Finally, in order to explain the movement of each IMF (residue) to the total movement of stock prices, we computed the variance and variance percentage of each IMF (residue). The results obtained for each of these measures are presented in Table 1.

We observed from Table 1 that, the residue which is known as the deterministic long-term trend by Huang et al. [6] was the dominant mode. It had the highest correlation coefficient with the observed prices of 0.9764 and 0.7769 for Pearson and Kendall correlation coefficients, respectively. The next was IMF6 with a mean period of 121.4. The residue contributed $94.9 \%$ of the total variation. For IMF1, IMF2, IMF3, IMF4, and IMF5, the values of the correlation coefficients were very low. These IMFs (IMF1-IMF5) only accounted for $1.39 \%$ of the total variation. This shows that the effect of (IMF1-IMF5) on stock prices was very low.

Next, following Zhu et al. [20], we used the hierarchical clustering and the Euclidean distance between pairs of any two IMFs or between an IMF and the residue to group the IMFs into three components. The clustering results are shown in Figure 3. When the Euclidean distance was less than 10, an IMF was classified as a high-frequency component, when the Euclidean distance was between 10 and 20, an IMF was classified as a lowfrequency component, and an IMF with a Euclidean distance of more than 20 was classified as the trend component. Therefore, IMF1-IMF5 were grouped as high-frequency component, IMF6 as the low-frequency component, and the residue served as the trend component. A plot of the extracted components and their statistical measures are presented in Figure 4 and Table 2, respectively.

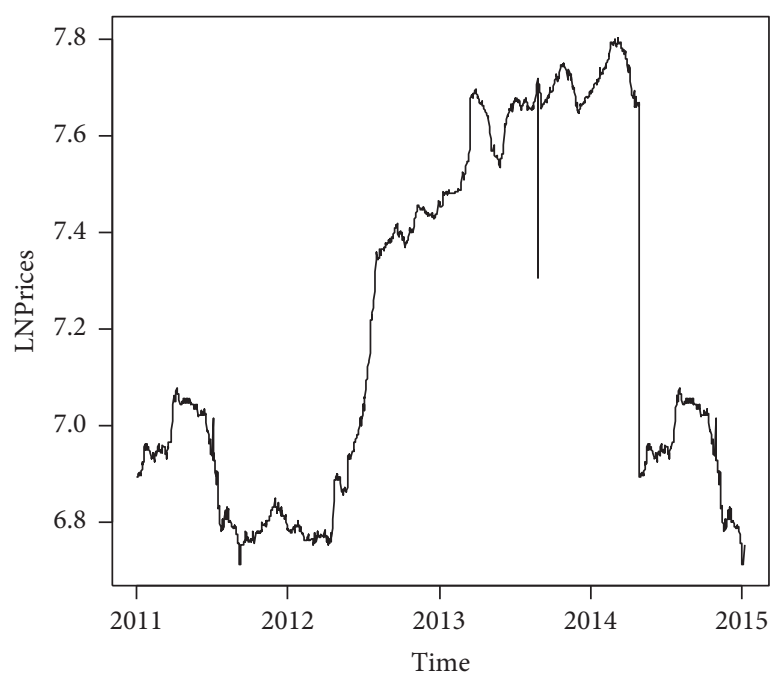

FIGURE 1: Plot of natural logarithmic values of GSEFSII from 04/01/ 2011 to $28 / 08 / 2015$.

Each of the 3 components in Figure 4 had distinct features. The residue varied around the long-term trend slowly. The residue was as thus considered as the longterm trend of stock prices. The correlation between the trend and stock prices contributed to about $96 \%$ of the total variation of the stock prices. The trend affected the long-term movement of stock prices in the long-run.

The high-frequency component reflected the effect of short-term market fluctuations such as speculation. Market fluctuations were short-lived and had little effect on stock prices as observed from the mean periods and variance values from Table 1.

The low-frequency component such as excessive government spending and the global financial crisis affected stock prices. An upward or downward movement indicated the effects of significant trend break on stock prices. The effect lasted longer than investors anticipated as evidenced by the mean period and variance values in Table 2.

The low frequency and trend components contributed to about $99.7 \%$ of the total variation and hence were considered as the most important components of the stock prices. Thus, the Nigerian stock exchange has driven real growth of the economy but not short-lived market fluctuations. Our results confirm the findings obtained in studies by Tiwari et al. [23] and Heaton and Lucas [36]. 

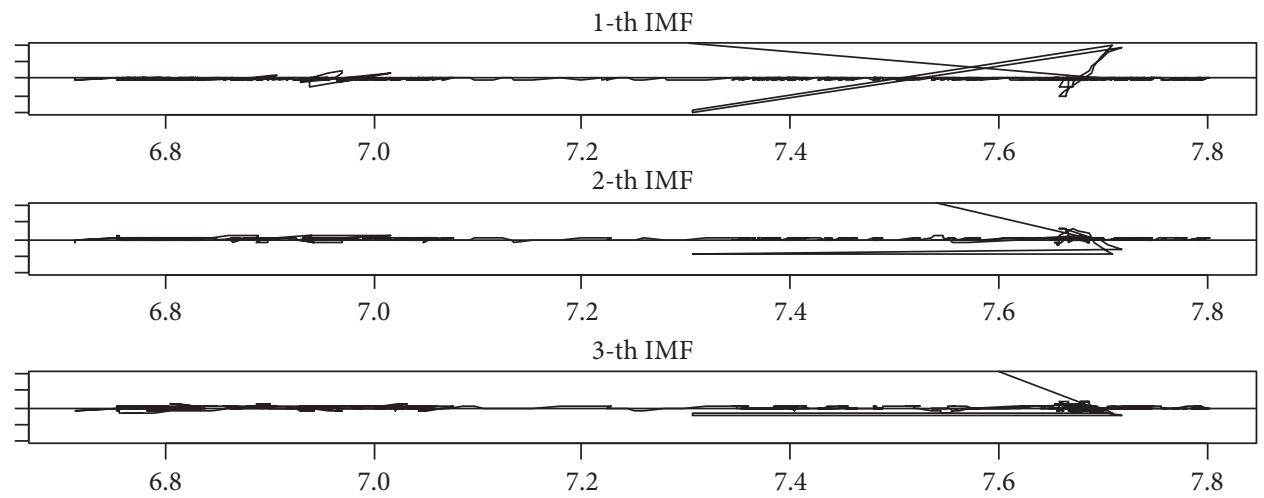

4-th IMF

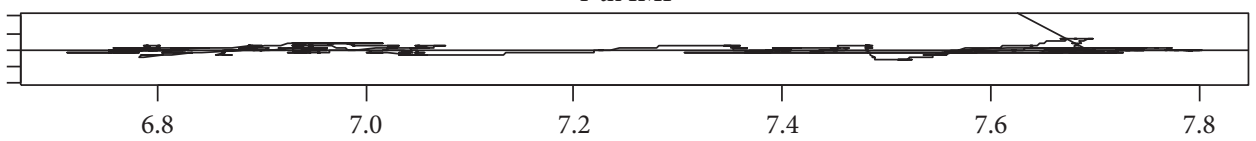

5-th IMF

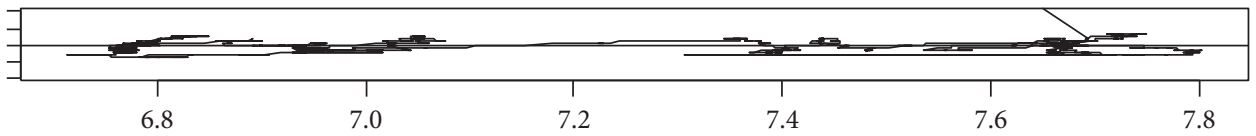

6-th IMF

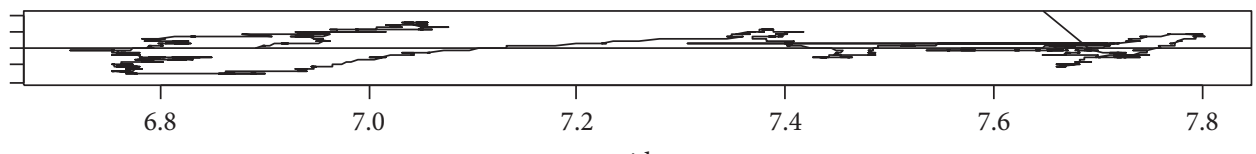

residue

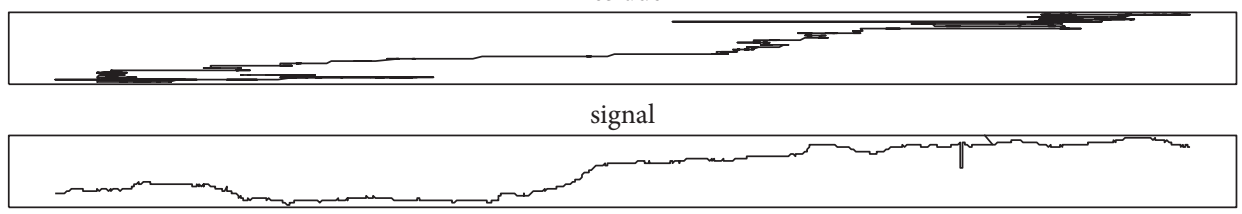

FIGURE 2: IMFs and residue of GSEFSI obtained using EMD.

TABLE 1: Measures of IMFs and residue of GSEFSII.

\begin{tabular}{|c|c|c|c|c|c|c|}
\hline & Mean period & Pearson correlation & Kendall correlation & Variance & Variance as $\%$ of observed & $\begin{array}{l}\text { Variance as \% of } \\
\sum \text { IMFs + residue }\end{array}$ \\
\hline Observed series & 2.3124 & & & 0.1404 & & \\
\hline IMF1 & 1.63 & 0.0288 & 0.0116 & 0.0003 & 0.20 & 0.20 \\
\hline IMF2 & 3.67 & 0.0211 & 0.0252 & 0.0001 & 0.07 & 0.07 \\
\hline IMF3 & 9.06 & 0.0133 & 0.0039 & 0.0001 & 0.07 & 0.07 \\
\hline IMF4 & 23.35 & $0.0937^{*}$ & $0.0711^{*}$ & 0.0004 & 0.28 & 0.28 \\
\hline IMF5 & 60.70 & 0.0471 & 0.0295 & 0.0011 & 0.77 & 0.77 \\
\hline IMF6 & 121.4 & $0.1824^{* *}$ & $0.1636^{* *}$ & 0.0052 & 3.70 & 3.71 \\
\hline Residue & 607 & $0.9764^{* *}$ & $0.7769^{* *}$ & 0.1330 & 94.73 & 94.90 \\
\hline \multicolumn{5}{|c|}{ Sum } & 99.82 & 100 \\
\hline
\end{tabular}

Note. ${ }^{*}$ and ${ }^{* *}$ : correlation is significant at the level of 0.05 and 0.01 (2-tailed) respectively. 


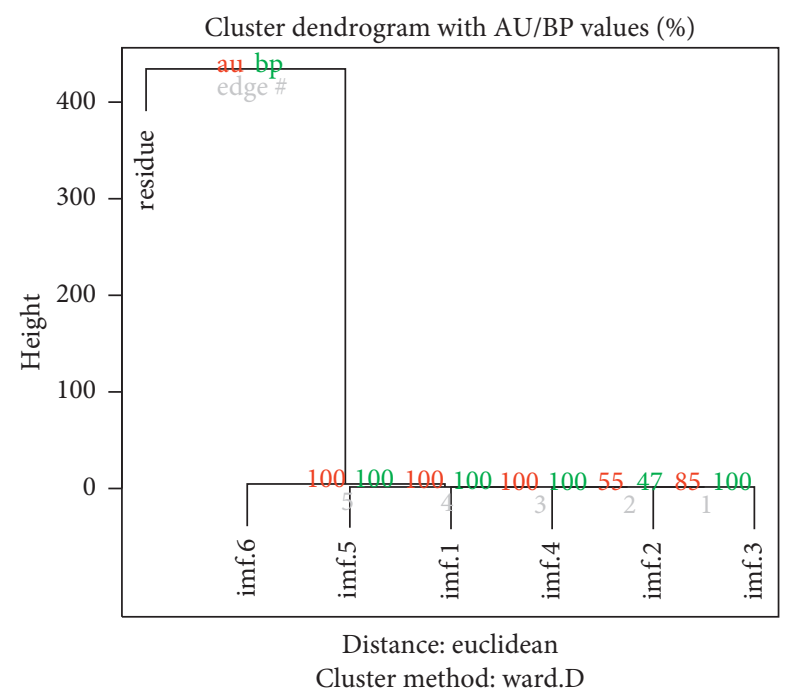

Figure 3: Hierarchical clustering tree obtained for the IMFs and residue.

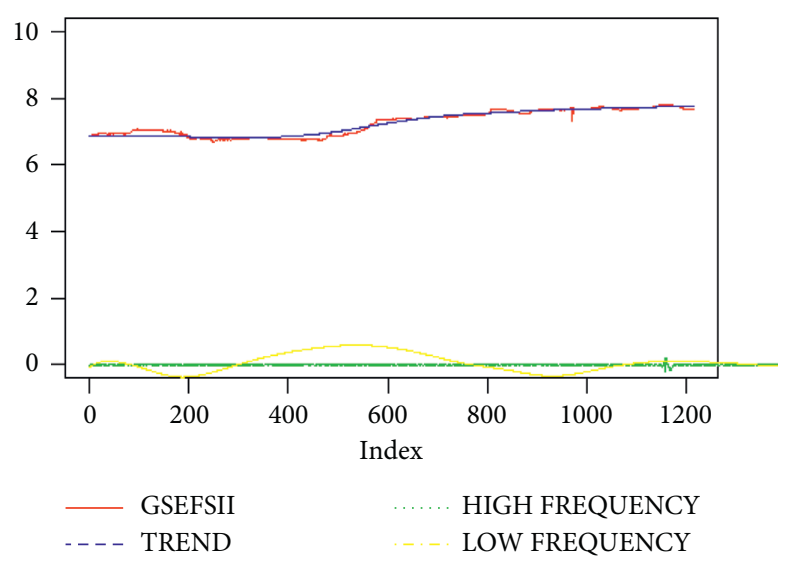

FIgURE 4: Three components of GSEFSII.

TABLE 2: Correlation and variance of components for GSEFII.

\begin{tabular}{lcccccc}
\hline & Mean period & Pearson correlation & Kendall correlation & Variance & Variance as \% of observed & $\begin{array}{c}\text { Variance as \% of } \\
\text { IMFs + residue }\end{array}$ \\
\hline Observed series & 2.3124 & & & 0.1401 & & \\
High Frequency & 4.7201 & 0.0421 & 0.0276 & 0.0004 & 0.2849 & 3.7037 \\
Low Frequency & 121.4 & 0.1824 & 0.1636 & 0.0052 & 94.73 & 9.7518 \\
Residue & 607 & $0.9764^{* *}$ & $0.7769^{* *}$ & 0.1330 & 98.7186 \\
\hline
\end{tabular}

${ }^{* *}$ Correlation is significant at the level of 0.05 (2-tailed).

\section{Conclusion}

The empirical mode decomposition (EMD) was used to decompose the natural logarithmic values of the daily closing prices of the GSE Financial Stock (GSE-FSI) index between 04/ 01/2011 and 28/08/2015 into six IMFs and a residue. Statistical measures such as the mean period, Pearson product moment correlation coefficient, and the Kendall rank correlation coefficient were used in assessing the effects of the IMFs and the residue on stock prices. The use of hierarchical clustering and Euclidean distance techniques helped us group IMFs and residue into high frequency, low frequency, and trend components. The trend and low-frequency components constituted about $99.7 \%$ of the total variation of the stock prices and were thus considered to affect stock prices than the high-frequency components. 
We, therefore, conclude that stock prices on the Ghana stock exchange are affected by real economic growth but not short-lived market fluctuations.

\section{Data Availability}

The data used were obtained from Thomson Reuters DataStream.

\section{Conflicts of Interest}

The authors declare that they have no conflicts of interest.

\section{References}

[1] T. Jin, H. Ding, B. Li, H. Xia, and C. Xue, "Valuation of interest rate ceiling and floor based on the uncertain fractional differential equation in Caputo sense," Journal of Intelligent \& Fuzzy Systems, vol. 40, no. 3, pp. 5197-5206, 2021.

[2] K. He, R. Zha, J. Wu, and K. Lai, "Multivariate EMD-based modeling and forecasting of crude oil price," Sustainability, vol. 8 , no. 4, p. 387, 2016.

[3] N. Nava, T. Matteo, and T. Aste, "Financial time series forecasting using empirical mode decomposition and support vector regression," Risks, vol. 6, no. 7, pp. 1-21, 2018.

[4] X. Qiu, H. Zhu, P. N. Suganthan, and G. A. J. Amaratunga, "Stock price forecasting with empirical mode decomposition based ensemble $v$-support vector regression model," in Computational Intelligence, Communications, and Business Analytics. CICBA 2017. Communications in Computer and Information Science, J. Mandal, P. Dutta, and S. Mukhopadhyay, Eds., Springer, Singapore, 2017.

[5] A. M. Adam and P. Owusu Junior, Financial Econometrics: An Example-Based Handbook, Nova Science Publishers, New York, NY, USA, 2017.

[6] N. E. Huang, Z. Shen, S. R. Long et al., "The empirical mode decomposition and the Hilbert spectrum for nonlinear and non-stationary time series analysis," Proceedings of the Royal Society of London. Series A: Mathematical, Physical and Engineering Sciences, vol. 454, no. 1971, pp. 903-995, 1998.

[7] L. Zhao, J. Gong, and H. Chong, "The extraction method of gearbox compound fault features based on EEMD and cloud model," Mathematical Problems in Engineering, vol. 2020, Article ID 6661975, 8 pages, 2020.

[8] H. Zhao, H. Liu, Y. Jin, X. Dang, and W. Deng, "Feature extraction for data-driven remaining useful life prediction of rolling bearings," IEEE Transactions on Instrumentation and Measurement, vol. 70, pp. 1-10, 2021.

[9] L. Yu, S. Wang, and K. K. Lai, "Forecasting crude oil price with an EMD-based neural network ensemble learning paradigm," Energy Economics, vol. 30, no. 5, pp. 2623-2635, 2008.

[10] X. Zhang, K. K. Lai, and S.-Y. Wang, "A new approach for crude oil price analysis based on empirical mode decomposition," Energy Economics, vol. 30, no. 3, pp. 905-918, 2008.

[11] X. Zhang, L. Yu, S. Wang, and K. K. Lai, "Estimating the impact of extreme events on crude oil price: an EMD-based event analysis method," Energy Economics, vol. 31, no. 5, pp. 768-778, 2009.

[12] C.-S. Lin, S.-H. Chiu, and T.-Y. Lin, "Empirical mode decomposition-based least squares support vector regression for foreign exchange rate forecasting," Economic Modelling, vol. 29, no. 6, pp. 2583-2590, 2012.
[13] B. Premanode and C. Toumazou, "Improving prediction of exchange rates using Differential EMD," Expert Systems with Applications, vol. 40, no. 1, pp. 377-384, 2013.

[14] V. Plakandaras, T. Papadimitriou, and P. Gogas, "Forecasting daily and monthly exchange rates with machine learning techniques," Journal of Forecasting, vol. 34, no. 7, pp. 560-573, 2015.

[15] A. M. Adam, K. Kyei, S. Moyo, R. Gill, and E. N. Gyamfi, "Similarities in Southern African Development Community (SADC) exchange rate markets structure: evidence from the ensemble empirical mode decomposition," Journal of African Business, pp. 1-16, 2021.

[16] N. An, W. Zhao, J. Wang, D. Shang, and E. Zhao, "Using multi-output feedforward neural network with empirical mode decomposition based signal filtering for electricity demand forecasting," Energy, vol. 49, pp. 279-288, 2013.

[17] F. Lisi and F. Nan, "Component estimation for electricity prices: procedures and comparisons," Energy Economics, vol. 44, pp. 143-159, 2014.

[18] Q. Hua and T. Jiang, "The prediction for London gold price: improved empirical mode decomposition," Applied Economics Letters, vol. 22, no. 17, pp. 1404-1408, 2015.

[19] P. Owusu Junior, A. M. Adam, and G. Tweneboah, "Connectedness of cryptocurrencies and gold returns: evidence from frequency-dependent quantile regressions," Cogent Economics \& Finance, vol. 8, no. 1, Article ID 1804037, 2020.

[20] B. Zhu, P. Wang, J. Chevallier, and Y. Wei, "Carbon price analysis using empirical mode decomposition," Computational Economics, vol. 45, no. 2, pp. 195-206, 2015.

[21] M. Xu, P. Shang, and A. Lin, "Cross-correlation analysis of stock markets using EMD and EEMD," Physica A: Statistical Mechanics and Its Applications, vol. 442, pp. 82-90, 2016.

[22] A. K. Tiwari, A. B. Dar, and N. Bhanja, "Oil price and exchange rates: a wavelet based analysis for India," Economic Modelling, vol. 31, no. 1, pp. 414-422, 2013a.

[23] A. Kumar Tiwari, A. Billah Dar, and N. Bhanja, "Stock market integration in Asian countries: evidence from wavelet multiple correlations," Journal of Economic Integration, vol. 28, no. 3, pp. 441-456, 2013 b.

[24] R. Jammazi, A. Lahiani, and D. Khuong, "Nguyen A waveletbased nonlinear ARDL model for assessing the exchange rate pass-through to crude oil prices," Journal of International Financial Markets, Institutions and Money, vol. 34, pp. 173187, 2015.

[25] C. Jiang, T. Chang, and X. L. Li, "Money growth and inflation in China: new evidence from a wavelet analysis," International Review of Economics \& Finance, vol. 35, pp. 249-261, 2015.

[26] R. Ferrer, V. J. Bolós, and R. Benítez, "Interest rate changes and stock returns: a European multi-country study with wavelets," International Review of Economics \& Finance, vol. 44, pp. 1-12, 2016.

[27] L. Yang, X. J. Cai, H. Zhang, and S. Hamori, "Interdependence of foreign exchange markets: a wavelet coherence analysis," Economic Modelling, vol. 55, pp. 6-14, 2016.

[28] G. J. Wang, X. Chi, and S. Chen, "Multiscale correlation networks analysis of the US stock market: a wavelet analysis," Journal of Economic Interaction and Coordination, vol. 12, no. 3, pp. 561-594, 2017.

[29] H. Boubaker and S. A. Raza, "A wavelet analysis of mean and volatility spillovers between oil and BRICS stock markets," Energy Economics, vol. 64, pp. 105-117, 2017.

[30] S. Frimpong, E. N. Gyamfi, Z. Ishaq, S. K. Agyei, D. Agyapong, and A. M. Adam, "Can global economic policy uncertainty drive the interdependence of agricultural commodity prices? 
Evidence from partial wavelet coherence analysis," Complexity, vol. 2021, Article ID 8848424, 13 pages, 2021.

[31] D. B. Percival and A. T. Walden, Wavelet Methods for Time Series Analysis, Volume 4 of Cambridge Series in Statistical and Probabilistic Mathematics, Cambridge University Press, Cambridge, UK, 2000.

[32] B. Wang, Y. M. Hu, Y. D. Du, Z. H. Zhai, and X. Y. Wu, "Study on the difference between wavelet analysis and EEMD in multi-scale decomposition of temperature and precipitation of Guangzhou," Journal of Tropical Meteorology, vol. 30, no. 4, pp. 769-776, 2014.

[33] J. Sun and H. Sheng, "Applications of ensemble empirical mode decomposition to stock-futures basis analysis," in Proceedings of the Information and Financial Engineering (ICIFE), 2010 2nd IEEE International Conference on, pp. 396-399, Chongqing, September 2010.

[34] H. Yu and H. Liu, "Improved stock market prediction by combining support vector machine and empirical mode decomposition," in Proceedings of the Computational Intelligence and Design (ISCID), 2012 Fifth International Symposium on, pp. 531-534, Hangzhou, October 2012.

[35] C. H. Cheng and L. Y. Wei, "A novel time-series model based on empirical mode decomposition for forecasting TAIEX," Economic Modelling, vol. 36, pp. 136-141, 2014.

[36] D. Lucas and J. Heaton, "Portfolio choice in the presence of background risk," Economic Journal, vol. 110, pp. 1-26, 2000. 